\title{
The Optimal Allocation of Power in Organizations
}

\author{
Emanuela Carbonara*† \\ Wadham College, University of Oxford \\ and \\ Dipartimento di Scienze Economiche, Università di Bologna
}

December 1999

\begin{abstract}
Starting from the separation between formal and real authority, the paper considers a hierarchical relation where delegation of control can be used as an incentive mechanism. It shows that delegation is optimal when parties' interests are neither too divergent nor too close. In that case superiors do not need to monitor too closely, curbing the subordinates' initiative and subordinates have no incentives to free ride on the superiors' information. The analysis is then extended to a multiperiod setting. If subordinates acquire expertise on the job (learning by doing), effort has the nature of a specific investment whose intrinsic value is higher the longer the subordinate's working life. Therefore, a policy of gradual delegation is possible, where agents accept weaker incentives at the beginning of their career, requiring stronger incentives over time as the value of their knowledge diminishes.

Keywords: Control Rights, Learning by Doing, Formal Authority, Real Authority, Dynamic Incentives.

JEL classification numbers: D23, L22, L23.
\end{abstract}

*Address for correspondence: Dipartimento di Scienze Economiche, 2, piazza Scaravilli, 40126 Bologna, ITALY. e-mail: carbonara@economia.unibo.it.

${ }^{\dagger}$ I am grateful to Philippe Aghion and Alan Beggs for their advice and encouragement. Carlo Scarpa provided valuable insights. I thank Piero Tedeschi for helpful comments on an earlier version. 


\section{Introduction}

An important issue in the literature on delegation and control in hierarchies is how power has to be shared between the top layer (principal, she) and subordinates (agents, he). We argue that, contrary to common wisdom, the principal may prefer not to delegate power to a subordinate when the subordinate's interests are too similar to the principal's ones. We then investigate the optimal dynamic pattern of delegation, showing that it is characterized by an increase in the amount of power held by a subordinate over time.

Following Aghion and Tirole (1997), we define formal authority the legal ability to make decisions in unforeseen circumstances or to control an asset, where the term legal implies that the attribution of authority is ratified by a contract. Conversely, real authority is the ability to exert actual control of the asset, authority conferred by the knowledge of important information. In general, formal does not imply necessarily real authority, especially in the presence of incomplete and asymmetric information. Often in organizations the individuals who hold formal authority are not the ones who take relevant decisions.

In this paper we analyze a situation where the principal and one agent perform several tasks, that differ in the degree of congruence of the parties' interests. For some tasks interests are very close and both parties would choose the same actions when free to decide. For other tasks, it is very unlikely that the most preferred options for one party coincide with the second party's ones. We investigate which activities the principal decides to delegate, given the divergence in interests. We also study how many out of these activities the principal delegates and what are the principles informing the extent of delegation.

As Aghion and Tirole (1997) show, delegating formal authority to an agent is both an incentive device to increase his initiative and a means to ensure his participation. After delegation, the principal no longer has the opportunity to overrule the agent and the agent's expected return from effort increases. ${ }^{1}$ That is why the agent's reservation utility is more easily achieved. Delegation, however, eliminates also the obstacles that prevent the agent from pursuing his own aims. The principal should then delegate formal authority only when the interests of both parties are close enough.

We show that, for given values of the parameters, the delegated activities may not be the ones characterized by the highest degree of coincidence of interests but rather those with "intermediate" congruence. To understand

${ }^{1}$ This result is termed basic-trade off between control and initiative by Aghion and Tirole (1997). 
such result, it should be noticed that, when congruence of interests is high, it is very likely that the agent achieves the principal's goals, even if motivated by self interest. This implies that the principal does not need to monitor him very closely, which enhances the agent's initiative, inducing high effort even if authority is not delegated. When parties' interests are very close, however, subordinates may have the incentive to shirk, free riding on the superior's knowledge and effort, even when they hold formal authority. ${ }^{2}$

We then investigate how the control structure of an organization is modified over time according to changes in the agent's ability. It is assumed that the agent's ability increases over time in a way that is proportional both to past effort exerted on a particular activity and to the number of times she has been performing that activity. We show that the agent is ready to spend high effort in earlier periods, when the cost reducing impact of effort lasts for a long time and the returns from a certain amount of effort are then large. The agent thus requires weaker incentives and works hard even if the principal delegates her a small number of activities. As time elapses and the agent's productive life approaches the end, the future benefits from effort are reduced and the principal needs to strengthen incentives, delegating more. We then reach the result, typical in the literature on dynamic incentives, that the intensity of incentives has to increase over time.

The nature of our result, however, differs from the traditional literature on incentives. In our case, it is neither because of career concerns nor because of implicit incentives that agents tend to put higher effort in the earlier stages of her contract. ${ }^{3}$ It is rather because his (specific) investment in effort is more remunerating that the agent is prone to work harder at the beginning of his career.

This paper is related to the literature on organizations and to the theory of contracts. Typically, the literature has stressed the incomplete nature of the contracts regulating the relationship between the owners (or, more generally, the top of the hierarchy of an organization) and subordinates. A contract has to specify clearly who has the power to decide in all contingent events not included in the contract.

Two main directions have been followed. On the one hand, it has been analyzed how a contract should allocate control rights over productive assets to a subordinate, especially when these assets (which can be both physical assets or human capital) are the result of specific investments, subject to the threat of hold-up problems (see Grossman and Hart, 1986 and Hart and

${ }^{2}$ This problem of free-riding is quite relevant and is usually overlooked in the typical literature on delegation and totally absent in Aghion and Tirole's (1997) analysis.

${ }^{3}$ For problems of dynamic incentives and for career concerns see Gibbons and Murphy (1982), MacLeod and Malcomson (1988) and Meyer and Vickers (1997). 
Moore, 1990). On the other hand, it has been studied how an owner (or, more generally, a superior) should provide incentives to work hard and make the "right" decisions to a subordinate, when the latter has control powers (this is the typical literature on agency and delegation, see, for instance, Grossman and Hart, 1983 or Holmström and Milgrom, 1987). The approach á lá Grossman, Hart and Moore deals with the allocation of formal authority in the presence of specific investments. The principal-agent approach takes as given the delegation of authority to subordinates and deals instead with incentive problems and informational asymmetry.

Aghion and Tirole (1997) present a synthesis between the literature on agency and on dynamic (implicit) incentives and the incomplete-contracts approach á lá Grossman and Hart (1986). They consider explicitly the role of information and the existence of real authority, in addition to formal. In their paper, Aghion and Tirole do not deal with intertemporal issues nor examine formally the relationship between delegation and congruence of interests. In this paper we want to provide an answer to both questions.

The paper is organized as follows. In Section 2 the basic setting with multitasking is introduced. Section 3 investigates how the congruence of interests between the principal and the agent influences the allocation of formal authority. Section 4 characterises the optimal pattern of delegation, taking into account also the agent's incentive to free ride on the principal's effort. Empirical implications of our results are presented in Section 5. Section 6 extends the analysis to a generic T-period time horizon and introduces learning by doing for the agent. Section 7 presents conclusions and interpretation of the results and, again, some empirical evidence about the intertemporal case. Appendix A contains the proofs. Appendix B shows that the results obtained here with linear cost functions can be generalized to different functional forms.

\section{The Basic Setting}

\section{Structure of the organization}

Consider a simple two-tier relationship consisting of a principal and an agent. The principal (she) hires the agent (he) to work on several economic activities and ensures his participation to the contract by paying him a wage in line with that currently available in the market. The performed activities generate an income that accrues to both parties, as it will be clarified below.

Before these activities are undertaken, the principal allocates control power (formal authority) upon them. For each activity she decides whether 
to retain formal authority or to delegate it to the agent. When the principal keeps formal authority, she is in charge of the decision process relative to that activity and has the right to impose her choices to the agent. If the principal delegates formal authority, the agent is able to pick his most preferred options without being overruled, even if these options are opposite to the principal's objectives.

\section{Production}

The organization undertakes a continuum of activities. ${ }^{4}$ Each activity consists of selecting one project out of a set of possible projects whose payoffs are unknown ex ante to both parties. A set $J$ of projects is associated to activity $j$. Activity $j$ consists of selecting and implementing a project from its associated set $J$.

There are many examples of organizations whose productive activity could be described in this way. It could be the case of the Research \& Development division of a firm, or picture the situation of a manager who has to choose a new product and its marketing strategy.

\section{Projects}

A project set $J$ contains $n_{j}$ projects, with $n_{j} \geq 3$. Among all projects belonging to a given set $J$ only two are relevant. This means that they give non-negative payoffs either to the principal or to the agent or to both. ${ }^{5}$ All other projects in $J$ always yield a very negative payoff to both.

Of the two relevant projects, one gives the principal a positive benefit equal to $\pi>0$, one gives the agent a positive benefit $b>0$. If the two positive benefits are provided by the same project we say that there is congruence of interests. The two positive benefits can be associated to different projects. In this second scenario, one out of the two relevant projects yields $\pi>0$ to the principal and 0 to the agent, whereas the other project yields $b>0$ to

\footnotetext{
${ }^{4}$ This assumption is introduced for technical reasons and represents a convenient way of modeling a framework where a very large number of tasks have to be performed.

${ }^{5}$ To understand why the agent may earn a private benefit from the productive activity and why, in some cases, the principal earns no benefits when the agent does think about the following examples. The agent may be interested in performing an $\mathrm{R} \& \mathrm{D}$ project that is of great interest for him but not for the firm (for instance, the firm stipulates a contract with an academic researcher who is interested in theoretical aspects, less relevant for the firm). Buying a certain machine may be bad for the firm but may benefit the agent (he may receive a bribe from the seller of the machine or establish a fruitful contact with a prospective future employer).
} 
the agent and 0 to the principal. ${ }^{6}$

All activities then yield potentially the same payoffs to the parties but differ in the degree of the parties' interests. With probability $\alpha_{j}$, in fact, the same project yields the positive benefit both to the principal and the agent and their interests are congruent. The probability $\alpha_{j}$ represents a measure of interest congruence (or congruence parameter). With complementary probability $1-\alpha_{j}$ different projects give $\pi_{j}$ to the principal and $b$ to the agent. Interests are therefore divergent and the two parties, when in charge of the decision process, would like to choose different projects. The payoffs from the two relevant projects (labeled 1 and 2) are described in the following table

\begin{tabular}{|c|c|c|}
\hline project 1 & project 2 & probability \\
\hline$\pi, b$ & 0,0 & $\alpha_{j}$ \\
\hline 0,0 & $\pi, b$ & $\alpha_{j}$ \\
\hline$\pi, 0$ & $0, b$ & $1-\alpha_{j}$ \\
\hline $0, b$ & $\pi, 0$ & $1-\alpha_{j}$ \\
\hline
\end{tabular}

Finally, because of the existence of very bad projects, a project will never be selected unless at least one of the parties knows for sure that the payoff is non-negative.

Activities are ordered by the principal in accordance to the congruence parameter $\alpha_{j}$ and can be represented on a segment of measure 1. Each activity is identified by its congruence parameter $\alpha_{j}{ }^{7}$ Together with the parameter $\alpha_{j}$, when deciding whether to delegate activity $j$, the principal takes into account the benefit she could potentially earn, $\pi$ and the agent's benefit $b$ (this last piece of information reveals how keen is the agent on the activity).

As already mentioned, the nature of the projects and their payoffs are unknown ex ante. A thorough process of screening is required in order to avoid these very bad projects and identify the relevant ones. Both the principal and the agent screen each set of projects. Exerting effort $e_{i}, i \in\{P, A\}$, $e_{i} \in\left\{\underline{e}_{i}, \bar{e}_{i}\right\}$, with $0 \leq \underline{e}_{i}<\bar{e}_{i} \leq 1$, party $i$ can learn the projects' true nature with probability $e_{i}$, whereas they remain uninformed with probability $1-e_{i}$. Thus, the more effort exerted, the higher the probability of finding out which is their preferred project.

\footnotetext{
${ }^{6}$ To abstract from problems of income distribution and rent extraction, $\pi$ and $b$ represent the monetary measure of benefits which are private in nature and cannot be expropriated.

${ }^{7}$ The choice of the congruence parameter as an ordering criterion is justified by the assumption that the principal considers the degree of congruence between her own and the agent's objectives as an indicator of the agent's reliability.
} 


\section{Timing}

The game consists of four stages and the timing is as follows. At stage one the principal decides the structure of control rights, allocating formal authority over certain activities to the agent. At stage two both parties collect information about all activities. At stage three, for each activity, the party with formal authority, if informed, chooses a project. If the party with formal authority is not informed, the other can exert real authority and suggest her most preferred project. ${ }^{8}$ The principal never overturns the agent's choice because she knows for sure that her payoff will be non-negative, i.e. the agent will never select a project yielding a very negative payoff. At stage four the selected projects are implemented and payoffs are realized. The timing of the model is summarized in Figure 1.

\section{Preferences and utility functions}

The principal is risk neutral. To abstract from problems of monetary incentives it is assumed that the agent is infinitely averse to income risk. ${ }^{9}$ The analysis focuses on the effects of the allocation of control on the agent's behavior and not on the design of optimal incentive schemes. This implies that he is not responsive to monetary incentives and receives a fixed payment equal to his reservation wage $\bar{w}$, which is normalized to zero to save on notation.

The principal and the agent maximize their expected payoffs from each activity $j$ with respect to $e_{P j}$ and $e_{A j}$, the effort they exert in that particular activity, $i=P, A$. Party $i$ learns the projects' payoffs with probability $e_{i}$, and is uninformed with probability $1-e_{i}$.

The principal's effort can take any value in the interval $[0,1]$. Her cost function is quadratic in effort and equal to $g_{P}\left(e_{P}\right)=k \frac{e_{P}^{2}}{2}$. The agent's effort can take only two values and it can be "high" or "low", $e_{A} \in\left\{\underline{e}_{A}, \bar{e}_{A}\right\}$. The agent's cost function is linear and equal to $g_{A}\left(e_{A}\right)=\gamma e_{A}$. The difference in the costs of screening, the principal's increasing faster, can be justified noticing that the principal might be in charge of more tasks than the agent. The latter performs only screening and project-selection functions, whereas

\footnotetext{
${ }^{8}$ In this model there is always a flow of information in both directions, from the principal to the agent and vice-versa. The agent makes suggestions to the principal when she is not informed about an activity she controls. Conversely, the principal communicates what she learned about the tasks on which the agent has been delegated formal authority.

${ }^{9}$ In making this assumption we follow Aghion and Tirole (1997). Technically, reasoning in terms of the linear incentive model by Holmström and Milgrom (1987), this implies that the term $\beta$ in the linear wage schedule $w=a+\beta x$ (where $w$ is the wage paid and $x$ is the agent's verifiable performance) is zero, so that the wage is fixed and equal to $a$.
} 
the principal has also to supervise and coordinate activities performed in other divisions and might experience work overload. ${ }^{10}$

Before introducing the model, it is important to clarify the specific concept of monitoring adopted here. It is monitoring ex ante: parties acquire information before action is taken and not after, to assess the results obtained. ${ }^{11}$ If the party with formal authority monitors and discovers the actions that suit her goals best, she overlooks any suggestion that might come from the second party, whose initiative and effort would then be reduced. That is why it is possible to identify a trade off between monitoring and initiative, trade off that does not exist if the traditional concept of monitoring is adopted.

\section{Principal's formal authority}

When the principal keeps formal authority over an activity, she overrules the agent -without taking her report into consideration- whenever she was able to find information herself. ${ }^{12}$ Only in cases where the principal has no information will the agent have real authority.

The principal's expected utility from an activity upon which she has formal authority is

$$
U_{P}\left(e_{P}, e_{A}, \pi, a\right)=e_{P} \pi+\left(1-e_{P}\right) e_{A} \alpha \pi-k \frac{e_{P}^{2}}{2}
$$

that is, with probability $e_{P}$ the principal is informed and therefore is able to choose and implement her preferred project, obtaining her private benefit $\pi$. The probability $e_{P}$ measures the extent to which the principal is able to exert both formal and real authority. Being informed gives her the real power to impose her formal authority. With probability $\left(1-e_{P}\right) e_{A} \alpha$ the principal is not informed whereas the agent obtained the information and chooses the same project.

The agent's expected payoff when the principal has formal authority is

$$
U_{A}\left(e_{A}, e_{P}, b, \alpha\right)=e_{P} \alpha b+\left(1-e_{P}\right) e_{A} b-\gamma e_{A}
$$

\footnotetext{
${ }^{10}$ For instance, the agent can be a division or an area manager, and the principal a superior, in charge of more than one division/area.

${ }^{11}$ Typically, the literature considers monitoring ex post or auditing. See, for instance, Baron and Besanko (1987).

${ }^{12}$ In this model information can be either hard (costlessly verifiable as soon as it is communicated to the principal) or soft (not verifiable), since this wouldn't modify the results.
} 
when the principal is informed (that happens with probability $e$ ), the agent obtains his private benefit $b$ only when interests are congruent. Conversely, when the principal is not informed and the agent is, he can exert real authority and choose his most preferred option. The probability $\left(1-e_{P}\right) e_{A}$ measures the extent of the agent's real authority. ${ }^{13}$

\section{Agent's formal authority}

When the agent holds formal authority over an activity, the expected payoffs are, respectively for the principal and the agent

$$
\widehat{U}_{P}\left(\hat{e}_{P}, \hat{e}_{A}, \pi, \alpha\right)=\widehat{e}_{A} \alpha \pi+\left(1-\widehat{e}_{A}\right) \widehat{e}_{P} \pi-k \frac{\widehat{e}_{P}^{2}}{2}
$$

the agent is informed with probability $\hat{e}_{A}$ and implements the project preferred by the principal with probability $\alpha$. With probability $\left(1-\widehat{e}_{A}\right) \widehat{e}_{P}$ the agent is not informed whereas the principal knows which the relevant projects are and can choose her preferred one. Similarly, the agent's payoff is

$$
\widehat{U}_{A}\left(\hat{e}_{A}, \hat{e}_{P}, b, \alpha\right)=\widehat{e}_{A} b+\left(1-\widehat{e}_{A}\right) \widehat{e}_{P} \alpha b-\gamma \widehat{e}_{A}
$$

when the agent is informed, he can pick his most preferred option. When the agent is not able to collect any information about that activity, whereas the principal knows this information, the agent's preferred project is chosen only if the principal's interests are the same as his, with probability $\alpha$.

\section{Allocation of Control and Congruence of Interests}

In this Section, the optimal allocation of control power according to interest congruence is analyzed. As mentioned above, several activities are performed in the organization. Specifically, there is a continuum of activities, each identified by its congruence parameter $\alpha$, where $\alpha \in[0,1]$ and is uniformly distributed over that interval.

\footnotetext{
${ }^{13}$ The cost per unit of effort is the same, no matter the activity such effort has been spent upon and who holds formal authority. This assumption is introduced to analyze the organizational structure that obtains when the leading criterion that guides the allocation of control is the congruence of interests and not differences in expertise and ability. For an analysis of delegation when the principal's costs change from one activity to another see Aghion and Tirole (1995).
} 
In order to write payoffs in a compact fashion, we can divide activities in two subsets according to the value of $\alpha$ that characterizes them. Call $D$ and $N D$ respectively the subsets of delegated and non-delegated activities. When activity $j$ is delegated, then $\alpha \in D$, when it is not delegated, then $\alpha \in N D$.

Given the structure of the payoffs described above, the principal chooses $e_{P}^{*}$ and $\widehat{e}_{P}^{*}$ in order to maximize ${ }^{14}$

$$
\begin{aligned}
& \int_{\alpha \in D}\left[e_{P} \pi+\left(1-e_{P}\right) e_{A} \alpha \pi-k \frac{e_{P}^{2}}{2}\right] f(\alpha) d \alpha+ \\
& +\int_{\alpha \in N D}\left[\widehat{e}_{A} \alpha \pi+\left(1-\widehat{e}_{A}\right) \widehat{e}_{P} \pi-k \frac{\widehat{e}_{P}^{2}}{2}\right] f(\alpha) d \alpha
\end{aligned}
$$

whereas the agent chooses $e_{A}^{*}$ and $\widehat{e}_{A}^{*}$ to maximize

$$
\int_{\alpha \in D}\left[e_{P} \alpha b+\left(1-e_{P}\right) e_{A} b-\gamma e_{A}\right] d \alpha+\int_{\alpha \in N D}\left[\widehat{e}_{A} b+\left(1-\widehat{e}_{A}\right) \widehat{e}_{P} \alpha b-\gamma \widehat{e}_{A}\right] d \alpha
$$

Both parties maximize their expected payoff from each activity in the continuum set $[0,1]$ and the level of effort chosen differs according to the distribution of control and the degree of interest congruence $\alpha$.

Notice that, following Aghion and Tirole (1995), in expressions (5) and (6) we are assuming cost separability, in the sense that effort spent on an activity does not influence costs borne working on other activities. Thus activities are neither complements nor substitutes. Each activity is completely different and working on it does not provide any useful expertise for the others. This assumption is made mainly to simplify the analysis, that otherwise would become formally intractable, unless specific assumption on the shape of the functionals expressing optimal efforts, $e_{P}^{*}$ and $e_{A}^{*}$ are made.

Cost separability has the nice feature that, since the problem has the same structure for each $\alpha \in D$ and for each $\alpha \in N D$, we can solve it pointwise, for each $\alpha \in D$ and $\alpha \in N D$.

\section{Principal's Formal Authority}

Given $\alpha \in A$, the problem for the principal is to maximize (1) with respect to $e_{P}$, while the agent chooses whether to exert high or low effort.

\footnotetext{
${ }^{14}$ The congruence parameter $\alpha$ is uniformly distributed on $[0,1]$. Its density is $f(\alpha)=1$. Indication of the density function will be omitted hereafter.
} 
From (1), the principal's optimal monitoring effort for any level of $e_{A}$ is

$$
e_{P}^{*}=\frac{\left(1-e_{A} \alpha\right) \pi}{k}
$$

Given that the agent's payoff is linear in $e_{A}$, he will exert high effort $\bar{e}_{A}$ as long as

$$
e_{P} \leq \frac{b-\gamma}{b}=e_{P}^{\lim }
$$

whenever the inequality in (8) is not satisfied, the agent exerts low effort $e_{A}=\underline{e}_{A}$.

\section{Agent's Formal Authority}

When the agent has formal authority over a certain activity, the expected payoffs are given by equation (3) and (4) respectively for the principal and the agent.

The principal's optimal monitoring for any level of $e_{A}$ is

$$
\widehat{e}_{P}^{*}=\frac{\left(1-\widehat{e}_{A}\right) \pi}{k}
$$

Notice that the principal's optimal effort with delegation does not depend on $\alpha$. This is because the principal can use her real authority, whose amount is directly proportional to her effort, only when the agent is uninformed. In that case, congruence of interests is irrelevant, as the principal can rely on her effort only and, if informed, can choose as she prefers.

Again, the agent's payoff is linear in effort, so that $\hat{e}_{A}=\bar{e}_{A}$ if and only if

$$
\widehat{e}_{P} \leq \frac{b-\gamma}{\alpha b}=\widehat{e}_{P}^{\lim }
$$

\section{Discussion of the results}

Comparing equations (8) and (10) leads to some interesting conclusions. The proofs for the Lemmas introduced below are immediate, recalling that $\alpha<1$.

Lemma 1. (Aghion and Tirole) The principal's effort in equilibrium is higher when she holds formal authority, $\widehat{e}_{P}^{*}<e_{P}^{*}$. 
This result is a consequence of the basic trade off between control and initiative. When the agent has formal authority over an activity, the principal has less incentives to bear high costs to monitor that activity, since she has less opportunities to use the information she might find.

Lemma 2. The maximum amount of monitoring an agent tolerates without being demotivated and ceasing to exert effort is higher when the agent holds formal authority, $\hat{e}_{P}^{\lim }>e_{P}^{\lim }$.

This second result is quite intuitive. If the agent has formal authority, he can pick his most preferred project whenever he is informed and the principal cannot stop him, even if she is informed and knows that the selected project is not her preferred one. Therefore, the agent knows that higher principal's effort in monitoring and hence a higher probability of acquiring information is counterbalanced by her lower ability to impose her preferences. The delegation of formal authority is a commitment device to increase the agent's initiative and it also allows a closer and more intense monitoring. ${ }^{15}$

\section{The delegation region}

The principal decides on the allocation of authority trying to keep control upon as many activities as she can. ${ }^{16}$ In order to characterize the optimal allocation of authority we need to prove the following result.

Proposition 1. There exist two values $\underline{\alpha}$ and $\bar{\alpha}$ for the congruence parameter $\alpha$ such that (a) if the principal wishes to induce $e_{A}=\bar{e}_{A}$, a necessary condition is to delegate those with $\alpha \in[\underline{\alpha}, \bar{\alpha}]$. (b) The principal keeps control over activities in the intervals $[0, \underline{\alpha})$ and $(\bar{\alpha}, 1]$ and, when $\alpha \in[0, \underline{\alpha})$, $e_{A}=\underline{e}_{A}$.

Proof. See Appendix.

It is interesting to see that the activities delegated are not the most congruent ones. Intuition would perhaps lead to conclude that the principal should loose control when the conflict of interests is not too sharp. On the other hand, the more congruent the activity, the lower the optimal level of monitoring and the easier it is for the principal to induce a high level of

\footnotetext{
${ }^{15}$ This could be extremely important when the principal wants to assess the agent's ability, for instance in order to promote him.

${ }^{16}$ This strategy relies on the presumption that, other things being held constant, the principal (weakly) prefers to keep formal authority rather that delegate it to subordinates. It is possible to show that such presumption is always correct.
} 
effort. There is no reason to delegate formal authority over such activities, given also that the cost of monitoring them is low.

It is also possible to show that this result is robust to changes in the shape of the cost function. Appendix B below provides an example where both parties have quadratic cost functions. We find that the principal retains control on activities with $\alpha>\bar{\alpha}$, where $\bar{\alpha}$ is the same value found in the case of linear costs.

Hereafter, the set of delegated activities will be called "delegation region".

Besides $\underline{\alpha}$ and $\bar{\alpha}$, there is another important cutoff value for $\alpha$, that characterizes the allocation of control, cutoff that has never been shown before in this kind of models. Define $\hat{\alpha}$ the value of the congruence parameter such that the agent is indifferent between exerting a low effort and working hard when he holds formal authority. In order to find $\hat{\alpha}$, recall that the agent prefers a high effort when the principal's monitoring on a delegated activity is low enough. This happens if $\widehat{e}_{P}^{*}=\widehat{e}_{P}^{\lim }$ and, from equations (9) and (10),

$$
\frac{\left(1-\bar{e}_{A}\right) \pi}{k}=\frac{b-\gamma}{\alpha b}
$$

Solving for $\alpha$

$$
\hat{\alpha}=\frac{b-\gamma}{b} \frac{k}{\left(1-\bar{e}_{A}\right) \pi}
$$

For $\alpha<\hat{\alpha}$, the principal's monitoring (which, on delegated activities, is constant and does not depend on $\alpha$ ) is low and the agent puts $e_{A}=\bar{e}_{A}$. If $\alpha>\hat{\alpha}$, instead, the agent puts no effort (thus free-riding on the principal's information), no matter that he has formal authority. Intuitively, both congruence and the principal's effort are high enough to guarantee that she will be informed and choose the right project. The agent has high probability to obtain his private benefit $b$ even with $e_{A}=\underline{e}_{A}$. Then, if the principal exerts enough effort, the agent free rides on her effort.

Given that the principal (weakly) prefers to keep control over an activity where $e_{A}=\underline{e}_{A}$, she does not delegate those with $\alpha>\hat{\alpha}$. Three cases have then to be distinguished.

Case 1. $\hat{\alpha}>\bar{\alpha}$. In this case, shown in Figure 2(a), the cutoff $\hat{\alpha}$ is not relevant $^{17}$ and the allocation of control is as described in Proposition 1.

Case 2. $\hat{\alpha}<\underline{\alpha}$. In this second case, shown in Figure 2(b), the whole delegation region disappears, as the agent exerts no effort in the range $[\underline{\alpha}, \bar{\alpha}]$ and there are no reasons to delegate him authority.

\footnotetext{
${ }^{17}$ The principal would delegate activities with $\alpha>\hat{\alpha}$ anyway.
} 
Case 3. $\underline{\alpha}<\hat{\alpha}<\bar{\alpha}$. The delegation region shrinks to $[\underline{\alpha}, \hat{\alpha}]$, whereas the principal retains control over activities with $\alpha \in[\hat{\alpha}, \bar{\alpha}]$, that, according to Proposition 1, were previously delegated. This last case is shown in Figure 2(c).

The following proposition describes the optimal allocation of control in an organization.

Proposition 2. If $\hat{\alpha}>\underline{\alpha}$, the Principal delegates control upon all activities with $\alpha \in\left[\underline{\alpha}, \alpha^{\lim }\right]$, where $\alpha^{\lim }=\min \{\hat{\alpha}, \bar{\alpha}\}$. If $\hat{\alpha}<\underline{\alpha}$, the principal never delegates control. Delegation would not induce the agent to work hard.

Two comparative-statics results then follow.

Corollary 1. When $\alpha^{\lim }=\hat{\alpha}$, the delegation region is smaller the higher $\pi$, the principal's private benefit. When $\alpha^{\lim }=\bar{\alpha}$, the delegation region shrinks with $\pi$ iff $\pi>\pi_{1}^{*}$, where $\pi_{1}^{*}=k\left[\frac{2(b-\gamma)}{\bar{b}_{A}\left(2-\bar{e}_{A}\right)}\right]^{\frac{1}{2}}$, otherwise, it becomes larger as $\pi$ increases.

Proof. See Appendix.

An implication of Corollary 1 is that, overall, the agent works less when the principal's private benefit $\pi$ is higher. A reduction in the delegation region implies that the agent exerts low effort on a larger number of activities. The principal prefers a less active worker when potential gains are high. ${ }^{18}$

Corollary 2. If $\pi$ is low enough, the delegation region increases as monitoring becomes easier for the principal (i.e. $k$ decreases).

Proof. See Appendix.

Corollary 2 shows the principal's attempt to solve the basic trade-off. When monitoring is easier, the principal tends to increase effort, thus weakening the agent's incentives to work hard. To restore them, the principal delegates more, if the expected loss implied by this policy is not too large.

Finally, a more able agent (characterized by a cost function with a lower $\gamma$ ) obtains formal authority upon a smaller share of activities, as the following Lemma states.

\footnotetext{
${ }^{18}$ If the principal's benefit $\pi<\pi^{*}$, the delegation region increases with $\pi$. This is because, as $\pi$ becomes larger, the principal's monitoring increases too (see equation (7)). It then becomes harder to satisfy the limit imposed by expression (8). Given that the principal's effort in decreasing in $\alpha$, the principal has to delegate activities with higher $\alpha$ in order to induce a high effort in the agent. If her private benefit is not too high $\left(\pi<\pi^{*}\right)$, the principal relinquishes more control rights rather than giving up on agent's effort.
} 
Lemma 3. When $\alpha^{\lim }=\bar{\alpha}$, an increase in $\gamma$ always enlarges the delegation region. When $\alpha^{\lim }=\hat{\alpha}$, an increase in $\gamma$ instead reduces delegation.

Proof. See Appendix.

This result is, again, intuitive. In this model, delegation occurs principally to induce the agent to work hard. If delegation is always an effective incentive device (which happens when $\hat{\alpha}>\bar{\alpha}$ and $\alpha^{\text {lim }}=\bar{\alpha}$ ) and the agent has low costs, it is easier to induce high effort and he tolerates high monitoring on a larger share of activities. The delegation region can thus be reduced. If instead the agent has a high propensity to free ride on the principal's effort and delegation is very likely to be ineffective $\left(\hat{\alpha}<\bar{\alpha}\right.$ and $\left.\alpha^{\lim }=\hat{\alpha}\right)$ an increase in costs, worsening the free-rider problem, induces the principal to reduce delegation.

The alternance of "intervals of congruence", where authority is not delegated, with intervals of delegation and the existence of activities where the principal tolerates the agent to exert no effort suggest a few interesting conclusions, summarized in the following proposition.

Proposition 3. The principal's effort is non monotonic in $\alpha$. It is highest on the least congruent activities, lowest on the delegation region and decreases with congruence on the most congruent activities. The agent's effort too is non monotonic in $\alpha$. It is high on the delegation region and on the nondelegated activities with highest $\alpha$. It is low on the least congruent activities and can alternate intervals of high and low effort if $\hat{\alpha}<\bar{\alpha}$.

The principal's and the agent's efforts when $\hat{\alpha}>\bar{\alpha}$ are depicted in Figures 3 (a) and 3(b) respectively, whereas the case with $\underline{\alpha}<\hat{\alpha}<\bar{\alpha}$ is depicted in Figures 4(a) and 4(b).

\section{Empirical implications}

The previous Sections have enlightened some criteria that influence (or should influence) the allocation of power in organizations. Lemma 2 predicts that agents with formal authority tolerate a higher degree of monitoring. An implication of this result is that more developed and efficient monitoring systems should be accompanied by higher degrees of delegation. This result is shown in Corollary 2, that states that, under given parameter constellations, lower monitoring costs for the principal (lower $k$ ) determine an enlargement of the delegation region.

A similar result can be found also in Burkart, Gromb and Panunzi (1997), who show that lower monitoring costs (hence more intense monitoring) lead 
to less concentrated ownership structures (that facilitate the manager's initiative). These results can be supported empirically by proving that ownership is more dispersed and the extent of delegation larger in environments that facilitate monitoring. There seems to be a strong relationship between the development of legal systems ${ }^{19}$ and ownership dispersion as Shleifer and Vishny (1997) show. More specifically, the result presented in Lemma 2 would be better supported by evidence about the relationship between monitoring technologies and the company's internal organization, especially the degree of decentralization and delegation. However, the studies cited above support the intuition that inside organizations there might be a concern not to reduce the manager's initiative.

The main result is Proposition 1, that states that the principal optimally delegates activities with "intermediate congruence", neither too low nor too high. Besides its intuitive implications, thoroughly presented above, this result seems difficult to test empirically. First of all, an objective measure of interest congruence should be defined, which looks like a hard task. ${ }^{20}$ It would also be necessary to define what it is meant by "intermediate" level of congruence.

Typically, higher layers in hierarchies tend to favor some employees, often independently of their abilities. The subjective criterion superiors use to evaluate employees may well be interest congruence. The tendency towards the creation of "yes men" ${ }^{21}$ seems to suggest that the favored employees present a high congruence of interests, as these employees are prone to follow the superior's preferences. This would not be enough, however, to reject the result in Proposition 1. Such evidence may be contaminated by various factors, overlooked intentionally in this analysis. Private benefits from an activity may be a decreasing function of interest congruence ${ }^{22}$ which is likely to shift preferences towards delegating most congruent activities. There may also be an upper constraint to the time and resources both the employee and her superior can dedicate to work. In that case, it can then happen that the most congruent activities are optimally delegated. An employee dedicates more time to delegated activities, where his real authority is then higher. If the principal cannot monitor as much as she liked because of time

\footnotetext{
${ }^{19}$ Especially the system's effectiveness in protecting minority investors and their ability to be informed about the company's performance).

${ }^{20} \mathrm{~A}$ possibility would be to interview a number of entrepreneurs and build an index based on their answers. This device, far from providing an objective evaluation, would also be affected by the same fragility of empirical results obtained by means of qualitative indices.

${ }^{21}$ See Prendergast and Stole (1996).

${ }^{22}$ Formally, $\pi=\pi(\alpha), \pi^{\prime}<0$.
} 
constraints, she would rather delegate most congruent activities, to better control relatively less congruent activities.

\section{The Dynamics of the Delegation of Power}

In this Section we want to extend the analysis to consider a multiperiod setting. We want to investigate whether and how it is optimal for the principal to change the allocation of authority over time and the extent of delegation.

\subsection{A necessary condition}

The agent has to screen the same set of activities indexed by the congruence parameter $\alpha \in[0,1]$ for $T$ periods. ${ }^{23}$ It is assumed that the agent learns over time how to perform better in his job. His learning is directly proportional to the total effort (current and past) exerted on each activity. To capture the idea of learning as an increase in the agent's skills and ability, it is assumed here that current effort spent on an activity reduces future marginal costs on that particular activity. Therefore effort becomes a sort of specific investment in skills, which has important incentive effects. This can be represented by means of the following cost function

$$
g_{A t}\left(e_{A 1}, e_{A 2}, \ldots, e_{A t}\right)=\gamma e_{A t}-l \sum_{i=1}^{t-1} e_{A i}
$$

where $\gamma$ and $l$ are scalars, $\gamma, l>0$. The parameter $\gamma$ represents the (constant) marginal cost in the initial period $t=1$. Given that the cost function (13) is linear, the agent's payoff too is linear in effort. ${ }^{24}$ According to (13), the effort spent working on an activity decreases the agent's marginal (and total) cost in all future periods by $l$ per effort unit. Thus, $l$ represents the rate (or speed) of learning.

As in Section 3, the total payoffs from the activities upon which the principal holds formal authority are, respectively for the principal and the

\footnotetext{
${ }^{23} \mathrm{~T}$ could be for example the duration of the life cycle of a given product and the agent has to choose the best marketing strategy for it. Alternatively, $T$ could be the working life of the agent himself.

${ }^{24}$ The analytical form for the cost function has been chosen following the traditional literature on learning by doing. According to that literature, learning causes a drop in production costs and the "cost function with learning" is usually decreasing with respect to cumulative production, so that learning is considered a type of economy of scale.
} 
agent

$$
U_{P}=\sum_{t=1}^{T} \delta_{P}^{t-1}\left\{\int_{\alpha \in N D}\left[e_{P t} \pi+\left(1-e_{P t}\right) e_{A t} \alpha \pi-\frac{k e_{P t}^{2}}{2}\right] d \alpha\right\}
$$

and

$$
U_{A}=\sum_{t=1}^{T} \delta_{A}^{t-1}\left\{\int_{\alpha \in N D}\left[e_{P t} \alpha b+\left(1-e_{P t}\right) e_{A t} b-\left(\gamma e_{A i}-l \sum_{i=1}^{t-1} e_{A i}\right)\right] d \alpha\right\}
$$

where $\delta_{x}, x \in\{P, A\}$, is the intertemporal discount factor for the principal and for the agent, $0<\delta_{x}<1$.

Conversely, the total payoffs for the activities delegated to the agent are, respectively for the principal and the agent

$$
\widehat{U}_{P}=\sum_{t=1}^{T} \delta_{P}^{t-1}\left\{\int_{\alpha \in D}\left[\widehat{e}_{A t} \alpha \pi+\left(1-\widehat{e}_{A t}\right) \widehat{e}_{P t} \pi-\frac{k \widehat{e}_{P t}^{2}}{2}\right] d \alpha\right\}
$$

and

$$
\widehat{U}_{A}=\sum_{t=1}^{T} \delta_{A}^{t-1}\left\{\int_{\alpha \in D}\left[\widehat{e}_{A t} b+\left(1-\widehat{e}_{A t}\right) \widehat{e}_{P t} \alpha b-\left(\gamma \hat{e}_{A t}-l \sum_{i=1}^{t-1} \widehat{e}_{A i}\right)\right] d \alpha\right\}
$$

In order to guarantee that the cost of effort is always non-negative, we assume that the following condition is always satisfied

$$
\gamma e_{A t}-l \sum_{j=1}^{t-1} e_{A j} \geq 0
$$

$\forall t$, where $t=1,2, \ldots ., T$. We thus have $T$ different conditions, one for each period $t$. Given that learning is directly proportional to the number of periods in which the agent performs a certain task, the $T$ conditions above can be replaced by the following inequality

$$
\gamma e_{A T}-l \sum_{j=1}^{T-1} e_{A j} \geq 0
$$


Given that the agent's cost function is linear in effort, he will always attain a corner solution, either exerting maximum effort $\bar{e}_{A}$ or no effort at all. If we consider that the agent can choose between two levels of effort only, $\underline{e}_{A}$ and $\bar{e}_{A}$, condition (19) becomes

$$
\gamma \underline{e}_{A}-l(T-1) \bar{e}_{A} \geq 0
$$

that is, costs are still positive if the agent has exerted high effort in all previous periods but low effort in $T$.

We now investigate under which conditions the agent exerts positive (maximum) effort $\bar{e}_{A}$. To solve this problem we proceed by backward induction.

For a given level of the agent's effort, the principal strictly prefers to keep formal authority upon an activity. In period $T$, the agent produces high effort $\left(e_{A T}=\bar{e}_{A}\right)$ in a non delegated activity if and only if the principal's monitoring level satisfies the following condition, which is obtained as condition (8) in Section 3

$$
e_{P T} \leq e_{P T}^{\lim }=\frac{b-\gamma}{b}
$$

It is immediate to check that the maximum amount of monitoring the agent tolerates in the last period is exactly the same as in the static model presented above. This happens because at time $T$ there will be no future benefits from exerting effort on an activity and we are therefore in a situation similar to a static game. Following the same procedure introduced in Proposition 1, it is possible to find $\bar{\alpha}_{T}$, the highest value of $\alpha$ that represents the upper limit to the delegation region at time $T$, that is, for any $\alpha>\bar{\alpha}_{T}$ the principal retains control. Given that $\bar{\alpha}_{T}=\bar{\alpha}$ and $\underline{\alpha}$ is invariant with respect to time and it is equal to the one found in the stating setting, ${ }^{25}$ the delegation region in period $T$ does not change when learning by doing is introduced.

We now proceed to calculate the maximum amount of monitoring and the limits to the delegation region for any period $t<T$. For all $t<T$ the problem presents the same structure. In a generic period $t$, if the principal holds formal authority, the agent will exert effort $e_{A t}=\bar{e}_{A}$ if and only if the principal's monitoring effort is lower than a certain threshold. The condition

\footnotetext{
${ }^{25}$ This can be checked directly from expression (31) in the proof to Lemma 3. The intuition for this result can be found considering that the principal's choice of whether to delegate and obtain high effort or not delegate and obtain low effort does not depend on the agent's costs. Therefore, the agent's decisions on how much effort to spend hence how much knowledge to acquire has no effect on the choice of $\underline{\alpha}$.
} 
on $e_{P}$ is obtained comparing the agent's choice between high and low effort in a certain period, given that in all following periods he will put high effort and has put high effort in all previous ones.

At period $t$ the agent faces the choice between exerting high or low effort, given the effort he spent in the past (that now represents his amount of knowledge) and the incentives he will face in future periods. Here we assume that in future periods incentives will be such that the agent will always put high effort but any alternative assumption would lead to the same results as the terms relative to future periods in the left and the right hand side cancel out. In fact, the effort exerted in a certain period $t$ does not influence the choice of effort in subsequent periods, effort that depends solely on the amount of monitoring the agent is subjected to in each period. ${ }^{26}$ The agent chooses to exert high effort in $t$ if and only if

$$
e_{P t} \leq e_{P t}^{\lim }=\frac{b-\gamma}{b}+\delta_{A} \frac{l}{b} \frac{1-\delta_{A}^{T-t-1}}{1-\delta_{A}}
$$

Equation (22) implies that the principal can optimally keep control over that activity while getting a high degree of effort from the subordinate if and only if $\alpha \geq \bar{\alpha}_{t}$, where $\bar{\alpha}_{t}$ is obtained as in Propositions 1 and is equal to

$$
\bar{\alpha}_{t}=\frac{1}{\bar{e}_{A}}-\frac{k\left[b-\gamma+\delta_{A} l \frac{1-\delta_{A}^{T-t-1}}{1-\delta_{A}}\right]}{\pi b \bar{e}_{A}}
$$

It is now possible to state the following result.

Lemma 4. The agent tolerates a decreasing level of monitoring ( $e_{P t-1}^{\lim }>$ $\left.e_{P t}^{\lim }\right)$ and the delegation region increases over time $\left(\bar{\alpha}_{t-1}<\bar{\alpha}_{t}\right)$, reaching its maximum extension at $t=T$.

Proof. Comparing the expressions for $e_{P t-1}^{\lim }$ and $e_{P t-1}^{\lim }$ obtained from (26) and for $\bar{\alpha}_{t-1}, \bar{\alpha}_{t}$ and $\bar{\alpha}_{T}$ from (23) and (32), it can be readily checked that $e_{P t-1}>e_{P t}$ and $\bar{\alpha}_{t}<\bar{\alpha}_{t-1}$ if and only if $\delta_{A}<1$. Then $e_{P T-1}^{\lim }>e_{P T}^{\lim }$ as $\delta_{A} \frac{l}{b} \frac{1-\delta_{A}^{T-t-1}}{1-\delta_{A}}>0$ and for the same reason $\bar{\alpha}_{T}<\bar{\alpha}_{T-1}$.

This last result has important implications. First of all, it states that the agent requires stronger incentives over time: notwithstanding the reduction

\footnotetext{
${ }^{26}$ The agent's choice of whether to put high or low effort in a certain period does not depend either on past or on future decisions and it is only a function of the intensity of monitoring, $e_{P t}$.
} 
in costs, he demands more delegation. The learning effect implies a reallocation of control rights from the principal to the agent period after period, in the sense that the delegation region becomes larger and larger as the agent learns. This is because the longer the time horizon faced by the agent (the lower $t$ ), the higher the number of periods in which the effort exerted in $t$ is going to reduce costs. Therefore, the agent needs weaker incentives to work hard, as the effort exerted today not only increases his real authority in the current period but also in all following periods. This explains why, in the last period $T$, only the effect on marginal costs matters. Effort exerted in $T$ influences only real authority in that period and has not future effects.

As time elapses, the period over which the agent can benefit from his investment in learning becomes shorter and he requires stronger incentives to work hard. To this purpose, the principal recurs to promotions and gives him formal authority over a larger number of activities. The result that an individual needs stronger incentives the closer to the end her working life, has been obtained also by Gibbons and Murphy (1992) although for completely different reasons. In their model, the principal has imperfect information about the agent's ability. In the early stages of his working life, the agent has the incentive to work harder in order to signal his ability and obtain better contractual terms in future periods. As the estimate of his ability becomes more and more precise in time, his career concerns lose strength and he needs more intense explicit incentives to work.

In our model, the agent's ability is known from start to both parties and the perspective of acquiring precious experience, useful for many periods to come, is the main reason for lighter incentives at the beginning. However, the problem of the agent free riding on the principal's effort exists also in a dynamic setting, as the following subSection will show.

\subsection{The dynamic adjustment}

We are now going to analyze for which values of $\alpha$ the agent exerts high effort $\bar{e}_{A}$ when he has formal authority. We are therefore looking for a cutoff equivalent to the value $\hat{\alpha}$ found in the static model and we want to see how such cutoff changes over time with the agent's learning. Again, to solve this problem, we proceed by backward induction.

In the final period $T$ there are no benefits from learning, as the game will not be repeated. From Section 3 we know that the agent exerts high effort $\bar{e}_{A}$ upon a delegated activity if both the principal's effort $e_{P}$ and congruence $\alpha$ are low enough not to provide sufficient guarantee that he will obtain his

private benefit $b$ in any case. Since the game at $T$ is analogous to a one-shot 
game, $\hat{e}_{A T}=\bar{e}_{A}$ if and only if

$$
\hat{e}_{P T} \leq \hat{e}_{P}^{\lim }=\frac{b-\gamma}{\alpha b}
$$

and this implies that $\hat{e}_{A T}=\bar{e}_{A}$ for any $\alpha \leq \hat{\alpha}_{T}$, where $\hat{\alpha}_{T}$ is obtained exactly as in Section 4 and is equal to

$$
\hat{\alpha}_{T}=\frac{b-\gamma}{b} \frac{k}{\left(1-\bar{e}_{A}\right) \pi}
$$

For any generic $t<T$, the agent puts high effort on a delegated activity if and only if

$$
\hat{e}_{P t} \leq \hat{e}_{P t}^{\lim }=\frac{b-\gamma}{\alpha b}+\delta_{A} \frac{l}{\alpha b} \frac{1-\delta_{A}^{T-t-1}}{1-\delta_{A}}
$$

At the optimum, the principal puts effort $\hat{e}_{P t}=\frac{1-\bar{e}_{A}}{k} \pi$, invariant with respect to $\alpha$. Equating (26) to $\hat{e}_{P t}$ and solving out for $\alpha$ yields the highest value of $\alpha$ that satisfies (26), $\hat{\alpha}_{t}$. For any $\alpha>\hat{\alpha}_{t}$ the agent free rides on the principal's effort and delegation is ineffective an incentive to exert effort. The value $\hat{\alpha}_{t}$ is

$$
\hat{\alpha}_{t}=\frac{k}{\left(1-\bar{e}_{A}\right) \pi}\left[\frac{b-\gamma}{b}+\delta_{A} \frac{l}{b} \frac{1-\delta_{A}^{T-t-1}}{1-\delta_{A}}\right]
$$

Lemma 5. The principal's effort level sufficient to provoke free riding decreases over time $\left(\hat{e}_{P t-1}>\hat{e}_{P t}^{\lim }\right)$ whereas the set of activities where the agent free rides increases over time $\left(\hat{\alpha}_{t}<\hat{\alpha}_{t-1}\right)$ and reaches a maximum at $T$.

Proof. Comparing the expressions for $\hat{e}_{P t-1}^{\lim }$ and $\hat{e}_{P t-1}^{\lim }$ obtained from (26) and for $\hat{\alpha}_{t-1}, \hat{\alpha}_{t}$ and $\hat{\alpha}_{T}$ from (27) and (25), it can be readily checked that $\hat{e}_{P t-1}>\hat{e}_{P t}$ and $\hat{\alpha}_{t}<\hat{\alpha}_{t-1}$ if and only if $\delta_{A}<1$. Moreover $\hat{e}_{P T-1}^{\lim }>\hat{e}_{P T}^{\lim }$ as $\delta_{A} \frac{l}{b} \frac{1-\delta_{A}^{T-t-1}}{1-\delta_{A}}>0$ and for the same reason $\hat{\alpha}_{T}<\hat{\alpha}_{T-1}$.

This result is in line with that in Lemma 5. As time elapses, the agent's specific investment becomes less and less valuable, because of the shorter and shorter time horizon. Hence he requires stronger incentives over time to put effort, even on delegated activities. This last finding adds to the characterization of the dynamics of control, dynamics that can now be fully described. Noticing that the value $\underline{\alpha}$ is invariant with respect to time, we can distinguish among three different cases, each one presenting some subcases. 
Case $1 \hat{\alpha}_{T}>\bar{\alpha}_{T}$. This case is equivalent to Case 1 in Section 4 . Here the cutoff $\hat{\alpha}_{t}$ is in the set of activities the principal never delegates at each $t$ and is therefore irrelevant. The dynamics of delegation is that described in Lemma 5, with the delegation region (and thus the strength of incentives) getting larger over time. This case is depicted in Figure 5(a).

Case $2 \hat{\alpha}_{T}<\underline{\alpha}$. This case is equivalent to Case 2 in Section 4 and three different subcases can be distinguished.

Case 2.a $\hat{\alpha}_{T}<\hat{\alpha}_{1}<\underline{\alpha}$. Here delegation never occurs. The agent exerts minimum effort $\underline{e}_{A}$ on all activities with $\alpha<\bar{\alpha}_{t}$ and the principal cannot induce more effort by delegation. The set of activities over which the agent puts $e_{A}=\bar{e}_{A}$ becomes smaller over time. This case is described in Figure 5(b).

Case 2.b $\hat{\alpha}_{T}<\underline{\alpha}<\bar{\alpha}_{1}<\hat{\alpha}_{1}$. Here $\bar{\alpha}_{1}$, the lower limit to the delegation region at $t=1$ and $\hat{\alpha}_{1}$, the cutoff value above which free riding occurs on delegated activities, move in opposite directions. Initially, $\bar{\alpha}_{1}<\hat{\alpha}_{1}$ and $\hat{\alpha}_{1}$ is irrelevant: the delegation region increases as in Case 1. At some time $t^{*}$ we have that $\hat{\alpha}_{t^{*}} \leq \bar{\alpha}_{t^{*}}$ and the agent starts to free ride on the principal's effort. His career concerns become too weak. If the principal based her incentive schemes only on delegation, then the span of delegation should decrease initially and then delegation should be abandoned altogether, when $\hat{\alpha}_{t}<\underline{\alpha}$. This is depicted in Figure 5(c).

Case 2.c $\hat{\alpha}_{T}<\underline{\alpha}<\hat{\alpha}_{1}<\bar{\alpha}_{1}$. In this case there is some delegation in earlier periods (the region $\left[\underline{\alpha}, \hat{\alpha}_{t}\right]$ as long as $\underline{\alpha}<\hat{\alpha}_{1}$ ). However, the delegation region shrinks over time and disappears at some $t^{*}$ such that $\hat{\alpha}_{t^{*}}<\underline{\alpha}$. Figure 5(d) illustrates this case.

Case $3 \underline{\alpha}<\hat{\alpha}_{T}<\bar{\alpha}_{T}$. The optimal dynamics of control in this case depends on the relative locations of $\bar{\alpha}_{1}$ and $\hat{\alpha}_{1}$. If $\bar{\alpha}_{1}<\hat{\alpha}_{1}$ we are in Case 2.b and the delegation region shrinks over time. If $\bar{\alpha}_{1}>\hat{\alpha}_{1}$ we are in Case 2.c and delegation never occurs.

\section{Concluding Remarks}

In this paper we have shown that the optimal allocation of control in an organization sees the principal keeping control over the least congruent activities (as we would expect) but also on the most congruent. Control is relinquished only on activities with an "intermediate" degree of congruence of interests. 
Moreover, delegation can be an effective way of motivating subordinates as long as the parties' interests are not too close. If this were the case, in fact, subordinates may try to free ride on the superior's effort even when they hold control powers.

Extending the model to an intertemporal setting, we reach a result similar to the ones typical of the literature on career concerns, where stronger incentives are needed over time. Here, we prove the need to increase the extent of the agent's authority and control over time and we provide a new rationale for internal careers and promotions.

In an attempt to test the relevance of career concerns, empirical studies have found little evidence of a link between managerial compensation and their performance. Jensen and Murphy (1990), for example, have shown that the sensitivity of CEO compensation to firm's value as a proxy for management productivity is very low.

We claim here that, more than career concerns, what drives the demand for stronger incentives is that, as the agent's working life carries on, on the one hand he acquires knowledge and on-the-job experience, which decreases his cost of effort. ${ }^{27}$ On the other hand, he is less and less interested in acquiring new knowledge that he would be able to exploit for a relatively short amount of time. High effort is thus obtained only at the expenses of more control.

In this sense, there is enough evidence of a positive correlation between compensation, power and experience, correlation our model is able to capture.

In our model, the demand for higher incentives is offset by an increasing tendency to free ride as time elapses. If this tendency is strong enough, delegation may prove useless as an incentive device. The role played by this free-riding effect in our model is even more important as we have intentionally ruled out reputation concerns, that would prevent an agent to shirk. However, also reputation concerns ${ }^{28}$ are likely to become weaker as a worker approaches the end of her career at which point her ability and skill should be generally known.

The problem of free riding in information collection has been studied also

\footnotetext{
${ }^{27}$ This decrease in effort costs can also be interpreted as an increase in the agent's market value due to the fact that his superior knowledge and experience make him attractive to other companies too.

${ }^{28}$ Reputation concerns are believed to be the main reason of the low correlation between pay and performance. A manager with reputation concerns is unwilling to undertake risky investments for fear of revealing scarce ability and in order to induce him to take risks, high pay may be required (see Holmström and Ricart I Costa, 1996 and Caruana and Celentani, 1999).
} 
by other contributions, mainly in the field of law and economics and jury decision making (e.g. see Davis, 1994 and Fedderson and Pesendorfer,1998) and the common feature in most of these contributions seems to be the public nature of information and the fact that the outcome obtained by using that information affects all parties equally. Information can be neither withheld nor misrepresented.

In our model, information assumes a strategic role not only because it is costly to acquire it but also because it can be used to favor one of the parties, possibly at the detriment of the others. There is a trade off between free riding and strategic manipulation of information. The more congruent the parties' interests the lower the strategic value of information and thus the more serious the free riding problem.

This stresses the importance of our result, that only activities with intermediate congruence ought to be delegated. On highly congruent activities, an agent is very likely to free ride.

\section{A Proofs}

\section{Proof of Proposition 1}

Step 1 The principal retains control over all activities with $\alpha>\bar{\alpha}$.

If the inequality in (8) holds, the principal optimally monitors according to $(7)$ and the agent puts high effort $e_{A}=\bar{e}_{A}$. When $e_{P}^{*}>e_{P}^{\lim }$, the inequality in (8) is violated and the agent puts low effort. If the principal wants high effort from the agent, the maximum monitoring she can exert is $e_{P}=e_{P}^{\lim }$. Otherwise, she can delegate to induce the high effort.

Since $e_{P}^{*}$ is decreasing in $\alpha$ and can take any value in the interval $\left[0, \bar{e}_{P}\right]$, there must exists an $\alpha$ s.t. $e_{P}^{*}=e_{P}^{\lim }$. Equating expressions (7) and (8) and solving for $\alpha$ yields

$$
\bar{\alpha}=\frac{1}{\bar{e}_{A}}-\frac{k(b-\gamma)}{\pi b \bar{e}_{A}}
$$

Since $e_{P}^{*}$ is decreasing in $\alpha, e_{P}^{*}<e_{P}^{\lim }$ for $\alpha>\bar{\alpha}$, the constraint in (8) is satisfied, the principal is maximizing her expected payoff and the agent puts high effort. There is no reason for the principal to delegate control.

If $\alpha<\bar{\alpha}, e_{P}^{*}>e_{P}^{\lim }$ and $e_{A}=0$.

The existence of $\bar{\alpha}$ is guaranteed by the conditions presented in the following Lemma. 
Lemma 6. (Existence and uniqueness of $\bar{\alpha}$ ). A relevant value $\bar{\alpha}$ exists (i.e. $0<\bar{\alpha}<1)$ iff $\left.\pi\right|_{\bar{\alpha}=0}<\pi<\left.\pi\right|_{\bar{\alpha}=1}$, where $\left.\pi\right|_{\bar{\alpha}=0}=\frac{k(b-\gamma)}{b},\left.\pi\right|_{\bar{\alpha}=1}=\frac{k(b-\gamma)}{b\left(1-\bar{e}_{A}\right)}$ and $\left.\pi\right|_{\bar{\alpha}=0}<\left.\pi\right|_{\bar{\alpha}=1}$.

Proof. The two thresholds $\left.\pi\right|_{\bar{\alpha}=0}$ and $\left.\pi\right|_{\bar{\alpha}=1}$ can be found immediately, respectively posing $\bar{\alpha}=0$ and $\bar{\alpha}=1$ and solving for $\pi$. Comparing the two values found as described shows that $\left.\pi\right|_{\bar{\alpha}=0}<\left.\pi\right|_{\bar{\alpha}=1}$.

The intuition for the result just stated is as follows. If the principal's benefit $\pi$ is very large $\left(\pi>\left.\pi\right|_{\bar{\alpha}=1}\right)$ then, $\forall \alpha, e_{P}^{*}>e_{P}^{\lim }$ and $e_{A}=0$. In equilibrium the principal wants to monitor very closely and this curbs the agent's initiative. If $\pi$ is very low $\left(\pi<\left.\pi\right|_{\bar{\alpha}=0}\right)$ then $\forall \alpha, e_{P}^{*}<e_{P}^{\lim }$ and $e_{A}=\bar{e}_{A}$. In equilibrium the principal does not monitor much and the agent has incentives to put high effort.

Assuming that a relevant value $\bar{\alpha}$ exists, for $\alpha<\bar{\alpha}$ the principal has two options.

a) Delegate activities with $\alpha<\bar{\alpha}$.

b) Hold formal authority on activities with $\alpha<\bar{\alpha}$, exert $e_{P}=e_{P}^{*}$ as given by (7) and have $e_{A}=0$.

Step 2 The principal delegates all activities with $\alpha \in[\underline{\alpha}, \bar{\alpha}]$, inducing the high effort $\bar{e}_{A}$ and holds formal authority, inducing the low effort 0 , on activities with $\alpha<\underline{\alpha}$.

The principal's payoff in case a) can be computed substituting her effort given by expression (9) into her payoff function (3), given $e_{A}=\bar{e}_{A}$

$$
\widehat{U}_{P}\left(\widehat{e}_{P}^{*}, \bar{e}_{A}, \pi, \alpha\right)=\bar{e}_{A} \alpha \pi+\frac{\left(1-\bar{e}_{A}\right)^{2} \pi^{2}}{2 k}
$$

whereas her payoff in case $b$ ) is obtained substituting effort as given by (8)

$$
U_{P}\left(e_{P}^{*}, 0, \pi, \alpha\right)=\frac{\pi^{2}}{2 k}
$$

Comparing the principal's payoffs in cases a) and $b$ ), it can be shown that $\widehat{U}_{P}\left(\widehat{e}_{P}^{*}, \bar{e}_{A}, \pi, \alpha\right)>U_{P}\left(e_{P}^{*}, 0, \pi, \alpha\right)$ if and only if $\alpha>\underline{\alpha}$, where

$$
\underline{\alpha}=\frac{\pi}{2 k}\left(2-\bar{e}_{A}\right)
$$

The existence of $\underline{\alpha}$ is guaranteed by the conditions presented in the following Lemma. 
Lemma 7. (Existence of $\underline{\alpha})$. A relevant value $\underline{\alpha}$ exists $(0<\underline{\alpha}<\bar{\alpha})$ iff $\pi_{1<\pi}<\pi_{2}$, where $\pi_{1}=-\pi_{2}=\frac{2 b k+k\left[b^{2}-2 b\left(2-\bar{e}_{A}\right) \bar{e}_{A}(b-\gamma)\right]^{\frac{1}{2}}}{b\left(2-\bar{e}_{A}\right) \bar{e}_{A}}$. The value $\underline{\alpha}$ is always positive.

Proof. Pose $\underline{\alpha}=\bar{\alpha}$ and solve for $\pi$. Equation $\underline{\alpha}=\bar{\alpha}$ is of second degree in $\pi$. Two solutions are then found, $\pi_{1,2}=\frac{2 b k \pm k\left[b^{2}-2 b\left(2-\bar{e}_{A}\right) \bar{e}_{A}(b-\gamma)\right]^{\frac{1}{2}}}{b\left(2-\bar{e}_{A}\right) \bar{e}_{A}}$. It can be checked that, in the admissible range for the parameters and given the existence of real roots, ${ }^{29}$ both $\pi_{1}$ and $\pi_{2}$ are positive and that $\underline{\alpha}-\bar{\alpha}<0$ iff $\pi_{1}<\pi<\pi_{2}$. From (31) it can be immediately seen that $\underline{\alpha}>0$.

These results are quite intuitive. If $\underline{\alpha}<0$, the principal would relinquish control of activity whose congruence is very small. This is not desirable. The principal prefers to keep control over all activities with $\alpha<\bar{\alpha}$, even if $e_{A}=0$, if her private benefit $\pi$ is very large $\left(\pi>\pi_{2}\right)$. In that case, $\underline{\alpha}>\bar{\alpha}$. The same happens if $\pi<\pi_{1}$. This second case would seem rather counterintuitive: the principal's benefit is very small and she does not delegate. This happens because, with such a small $\pi$, her optimal monitoring effort is very low and her real authority too little to delegate.

\section{Proof of Corollary 1}

$\alpha^{\lim }=\hat{\alpha}$ It is immediate to show that the threshold $\underline{\alpha}$ increases with $\pi$. Moreover, $\frac{\partial \hat{\alpha}}{\partial \pi}<0$. The delegation region $[\underline{\alpha}, \hat{\alpha}]$ becomes smaller as $\pi$ increases.

$\alpha^{\lim }=\bar{\alpha} \frac{\partial \bar{\alpha}}{\partial \pi}>0$, then the limits of the delegation region $[\underline{\alpha}, \bar{\alpha}]$ change in the same direction. If $\left|\frac{\partial \underline{\alpha}}{\partial \pi}\right|>\left|\frac{\partial \bar{\alpha}}{\partial \pi}\right|$, the delegation region moves toward more congruent activities and shrinks as $\pi$ increases. It can be checked that $\left|\frac{\partial \underline{\alpha}}{\partial \pi}\right|>\left|\frac{\partial \bar{\alpha}}{\partial \pi}\right|$ iff $\pi>\pi_{1}^{*}=k\left[\frac{2(b-\gamma)}{b \bar{e}_{A}\left(2-\bar{e}_{A}\right)}\right]^{\frac{1}{2}}$.

\section{Proof of Corollary 2}

First of all, check that $\underline{\alpha}$ increases as $k$ decreases (i.e. $\frac{\partial \alpha}{\partial k}<0$ ). When monitoring is less costly, the principal prefers to keep control over a larger portion of low-congruence activities.

$\alpha^{\lim }=\bar{\alpha}$ Check that $\bar{\alpha}$ increases as $k$ decreases $\left(\frac{\partial \bar{\alpha}}{\partial k}<0\right)$. Then, then the limits of the delegation region $[\underline{\alpha}, \bar{\alpha}]$ change in the same direction. If

\footnotetext{
${ }^{29}$ The equation $\underline{\alpha}-\bar{\alpha}=0$ has real roots iff $\left(1-4 \bar{e}_{A}+2 \bar{e}_{A}^{2}\right) b+2 \bar{e}_{A} \gamma\left(2-\bar{e}_{A}\right)>0$.
} 
$\left|\frac{\partial \alpha}{\partial k}\right|<\left|\frac{\partial \bar{\alpha}}{\partial k}\right|$, the delegation region moves toward more congruent activities and becomes larger as $k$ increases. The inequality $\left|\frac{\partial \alpha}{\partial k}\right|<\left|\frac{\partial \bar{\alpha}}{\partial k}\right|$ holds iff $\pi<\pi_{1}^{*}=k\left[\frac{2(b-\gamma)}{b \bar{e}_{A}\left(2-\bar{e}_{A}\right)}\right]^{\frac{1}{2}}$, where $\pi_{1}^{*}$ is the same value found in Corollary 1.

$\alpha^{\lim }=\hat{\alpha}$ The cutoff $\hat{\alpha}$ is decreasing in $k$. Then, then the limits of the delegation region $[\underline{\alpha}, \hat{\alpha}]$ change in the same direction. If $\left|\frac{\partial \underline{\alpha}}{\partial k}\right|<\left|\frac{\partial \hat{\alpha}}{\partial k}\right|$, the delegation region moves toward more congruent activities and becomes larger as $k$ increases. The inequality $\left|\frac{\partial \underline{\alpha}}{\partial k}\right|<\left|\frac{\partial \bar{\alpha}}{\partial k}\right|$ holds iff $\pi<\pi_{2}^{*}=k\left[\frac{2 k}{\left(2-\bar{e}_{A}\right)\left(1-\bar{e}_{A}\right) b}\right]^{\frac{1}{2}}$.

\section{Proof of Lemma 3}

It can be readily checked from (31) that the threshold $\underline{\alpha}$ is invariant with respect to $\gamma$. From (28), the cutoff $\bar{\alpha}$ is always increasing in $\gamma$. From (12), $\hat{\alpha}$ is always decreasing in $\gamma$.

\section{Proof of Lemma 4}

From the principal's payoff function (14) it can be checked that, if $e_{A T}=\bar{e}_{A}$, then $e_{P T}^{*}=\frac{\left(1-\alpha \bar{e}_{A}\right) \pi}{k}$. When the inequality in $(21)$ is satisfied and $e_{P T}^{*} \leq e_{P T}^{\lim }$, then $e_{A T}^{*}=\bar{e}_{A}$. There exist a value for $\alpha$ such that $e_{P T}^{*}=e_{P T}^{\lim }$. From the expression for $e_{P T}^{*}$ and (21) this cutoff is

$$
\bar{\alpha}_{T}=\frac{1}{\bar{e}_{A}}-\frac{k\left(b-\frac{\gamma}{T}\right)}{\pi b \bar{e}_{A}}
$$

and comparing expression (32) with (28) obtained in Proposition 1 it can be seen that $\bar{\alpha}_{T}<\bar{\alpha}$.

When $\alpha>\bar{\alpha}_{T}$, then $e_{P T}^{*}<e_{P T}^{\lim }$ and $e_{A T}^{*}=\bar{e}_{A}$. When $\alpha<\bar{\alpha}_{T}$, then $e_{P T}^{*}>e_{P T}^{\lim }$ and $e_{A T}^{*}=0$. If the principal wants to obtain high effort from the agent, she has to delegate.

From Proposition 1, there exist a cutoff $\underline{\alpha}$ such that the principal prefers to keep formal authority upon activities with $\alpha<\underline{\alpha}$, even if this implies $e_{A T}^{*}=0$. Given that the cutoff obtained in Proposition 1 does not depend on the agent's cost (see equation (31)), $\underline{\alpha}$ is the same in all periods and is not influenced by the agent's learning process. 


\section{B Delegation with quadratic agent's costs}

In this Appendix, we want to show that the result obtained in Section 4, that optimal delegation concerns activities with intermediate degree of congruence of interests, does not depend on the linear form chosen for the agent's cost function. In order to do this, we assume that the agent's costs are expressed by a quadratic cost function, equivalent to the principal's one, of the kind

$$
g_{A}\left(e_{A}\right)=\gamma \frac{e_{A}^{2}}{2}
$$

For a given activity with congruence $\alpha$, the agent's payoff function when then principal has formal authority and when authority is delegated to the agent are, respectively

$$
e_{P} \alpha b+\left(1-e_{P}\right) e_{A} b-\gamma \frac{e_{A}^{2}}{2}
$$

and

$$
\widehat{e}_{A} b+\left(1-\widehat{e}_{A}\right) \widehat{e}_{P} \alpha b-\gamma \frac{\widehat{e}_{A}^{2}}{2}
$$

The first order conditions are, respectively

$$
e_{A}=b \frac{1-e_{P}}{\gamma}
$$

and

$$
\hat{e}_{A}=b \frac{1-\alpha \hat{e}_{P}}{\gamma}
$$

Given the principal's first order conditions expressed in the main text by in (7) and (9), the equilibrium levels of effort are

$$
e_{A}^{*}=\frac{b(k-\pi)}{k \gamma-\alpha b \pi}, \quad e_{P}^{*}=\frac{\pi(\gamma-\alpha b)}{k \gamma-\alpha b \pi}
$$

and

$$
\hat{e}_{A}^{*}=\frac{b(k-\alpha \pi)}{k \gamma-\alpha b \pi}, \quad \hat{e}_{P}^{*}=\frac{\pi(\gamma-b)}{k \gamma-\alpha b \pi}
$$

From (38) it can be checked that $e_{A}^{*} \leq \bar{e}_{A}<1$ if and only if $\alpha \leq \bar{\alpha}$, where

$$
\bar{\alpha}=\frac{1}{\bar{e}_{A}}-\frac{k(b-\gamma)}{\bar{e}_{A} \pi b}
$$

which is exactly the same value found in Proposition 1 with a linear cost function. If $\alpha>\bar{\alpha}$, then $e_{A}^{*}=\bar{e}_{A}$ and there is no need to delegate. 


\section{References}

[1] Aghion, P. and J.Tirole, 1995, Some Implications of Growth for Organizational Form and Ownership Structure, European Economic Review, Papers and Proceedings.

[2] Aghion, P. and J.Tirole, 1997, Formal and real Authority in Organizations, Journal of Political Economy 105, 1-29.

[3] Baker, G., R. Gibbons and K.J. Murphy (1999), Informal Authority in Organizations, Journal of Law, Economics and Organization 15, 56-73.

[4] Baron, D. P. and D. Besanko, 1987, Monitoring, Moral Hazard, Asymmetric Information, and Risk Sharing in Procurement Contracting, Rand Journal of Economics 18, 509-32.

[5] Burkart, M., D.Gromb and F.Panunzi, 1997, Large Shareholders, Monitoring and the Value of the Firm, Quarterly Journal of Economics 112, 693-728.

[6] Caruana, G. and M. Celentani, 1999, Career Concerns, Incentive compensation and Investment Decisions, mimeo, Universidad Carlos III de Madrid.

[7] Cremer, J., 1995, Arm's Length Relationships, Quarterly Journal of Economics 110, 275-295.

[8] Davis, M., 1994, The Value of Truth and the Optimal Standard of Proof in Legal Disputes, Journal of Law, Economics and Organization 10, 343359.

[9] Fedderson, T. and W. Pesendorfer, 1998, Convicting the Innocent: The Inferiority of Unanimous Jury Verdicts under Strategic Voting", American Political Science Review 92, 23-35.

[10] Gibbons, R. and K.J. Murphy, 1992, Optimal Incentive Contracts in the Presence of Career Concerns: Theory and Evidence, Journal of Political Economy 50, 911- 930.

[11] Grossman, S.J. and O. Hart, 1983, An Analysis of the Principal-Agent Problem, Econometrica 51, 7-45.

[12] Grossman, S.J. and O. Hart, 1986, The Costs and Benefits of Ownership: a Theory of Vertical and Lateral Integration, Journal of Political Economy 94, 691- 719. 
[13] Hart, O. and J. Moore, 1990, Property Rights and the Nature of the Firm, Journal of Political Economy 98, 1119-58.

[14] Holmström, B. and P. Milgrom, 1987, Aggregation and Linearity in the Provision of Intertemporal Incentives, Econometrica 55, 303-28.

[15] Holmström, B. and P. Milgrom, 1991, Multi-task Principal Agent Analysis: Incentive Contracts, Asset Ownership and Job Design, Journal of Law, Economics and Organization 7, 26-52.

[16] Holström, B. and J. Ricart I Costa, 1986, Managerial Incentives and Capital Management, Quarterly Journal of Economics 101, 835-860.

[17] Jensen, M. and K. Murphy, 1990, Performance Pay and TopManagement Incentives, Journal of Political Economy 98, 225-264.

[18] MacLeod, W.B. and J. Malcomson, 1988, Reputation and Hierarchy in Dynamic Models of Employment, Journal of Political Economy 96, 832-854.

[19] Malcomson, J., 1984, Work Incentives, Hierarchy and Internal Labour Markets, Journal of Political Economy 92, 486-507.

[20] Meyer, M.A., 1994, The Dynamics of Learning with Team Production: Implications for Task Assignment, Quarterly Journal of Economics 109: $1157-1184$.

[21] Meyer, M.A. and J. Vickers, 1997, Performance Comparisons and Dynamic Incentives, Journal of Political Economy 105, 547-581.

[22] Prendergast C. and R.H. Topel (1996), Favoritism in Organizations, Journal of Political Economy, vol.104, no.5:958-978.

[23] Sah, R. and J.Stiglitz, 1986, The Architecture of Economic Systems: Hierarchies and Polyarchies, American Economic Review 76, 716-727.

[24] Shleifer A. and R.W. Vishny, 1997, A Survey of Corporate Governance, Journal of Finance 52, 737-83.

[25] Williamson, O.E., 1975, Market and Hierarchies: Analysis and Antitrust Implications (Free Press, New York). 


\section{Figure 1}

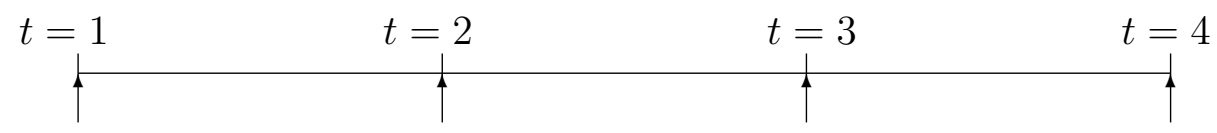

Principal allocates Information collected Information exchanged Projects implemented formal authority

Projects chosen

Payoffs realized

Figure 2(a)

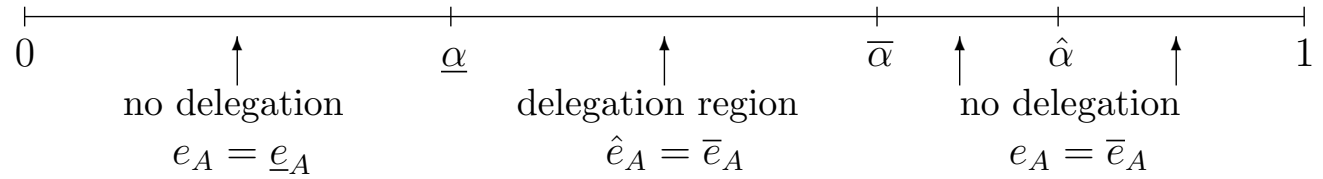

Figure 2(b)

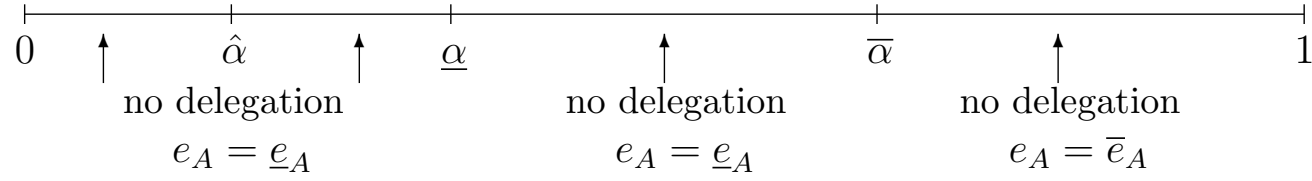

Figure 2(c)

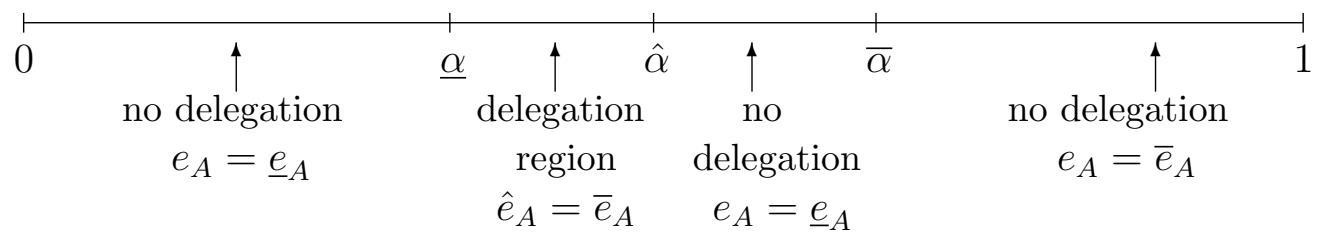


Figure 3(a)

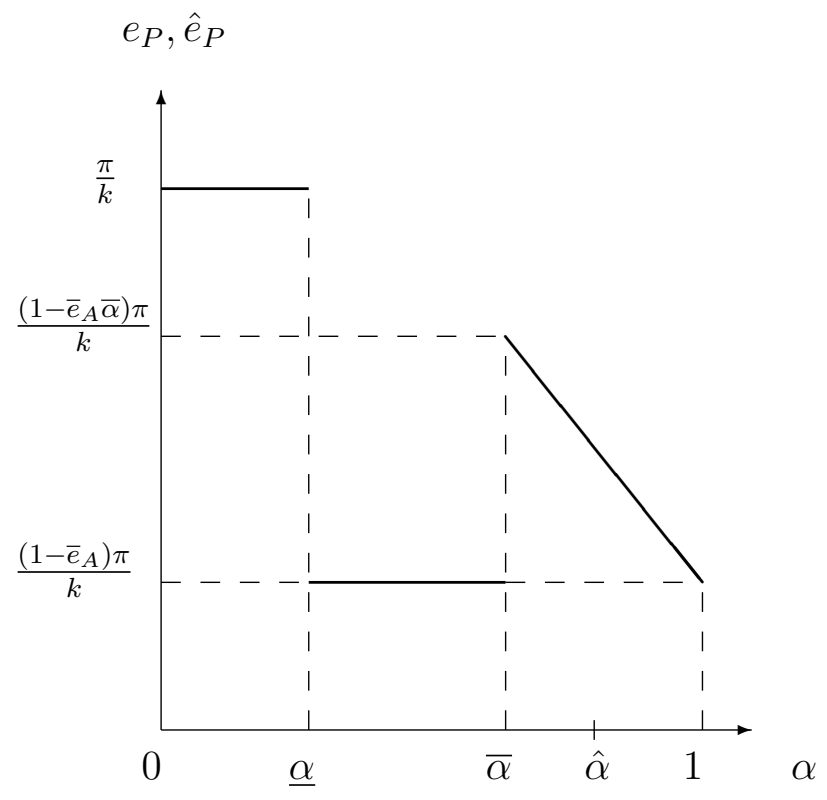

Figure 3(b)

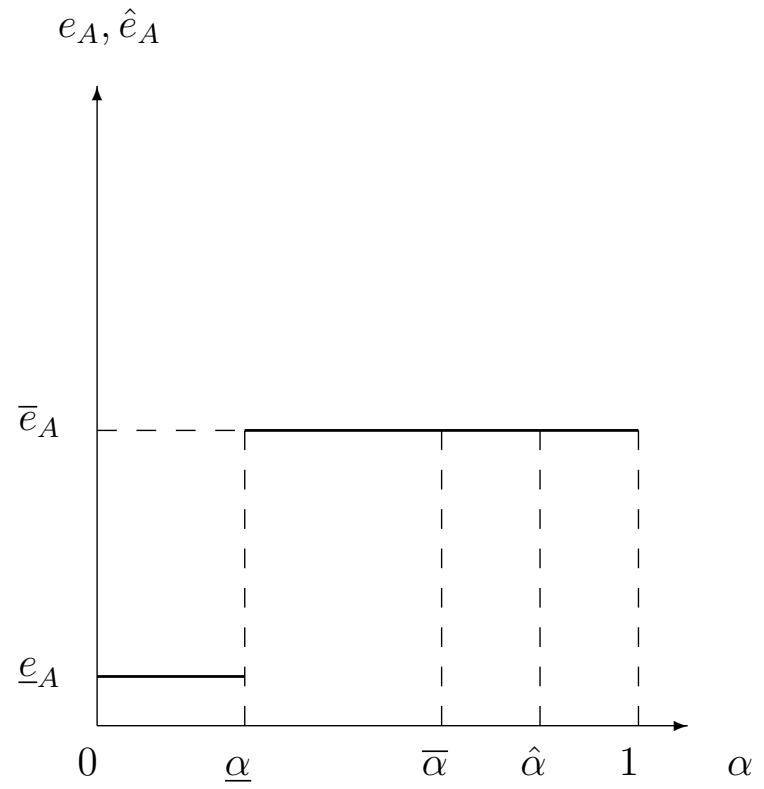


Figure 4(a)

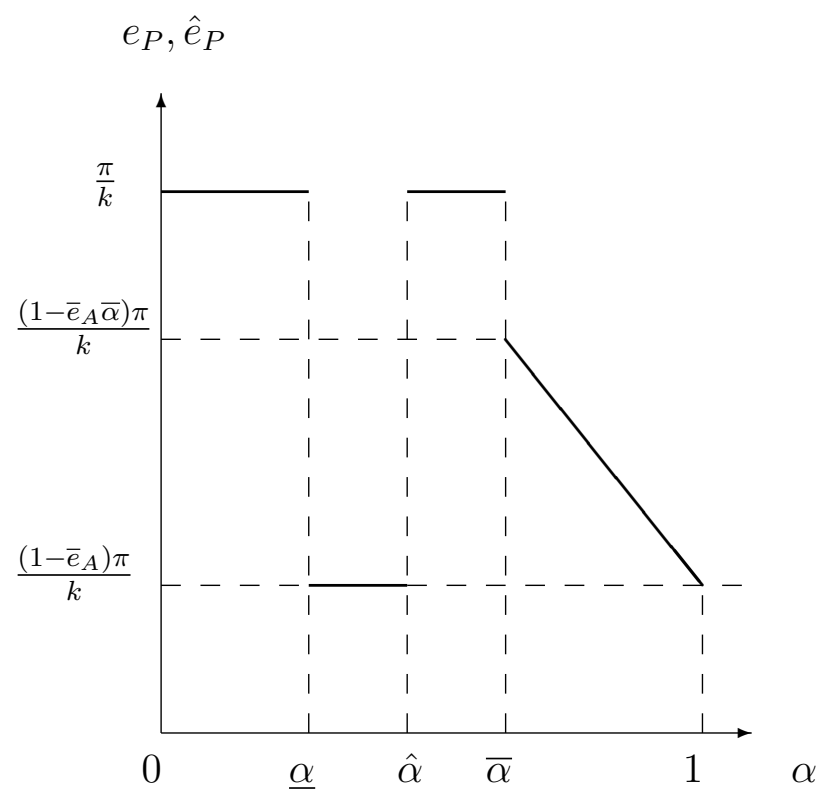

Figure 4(b)

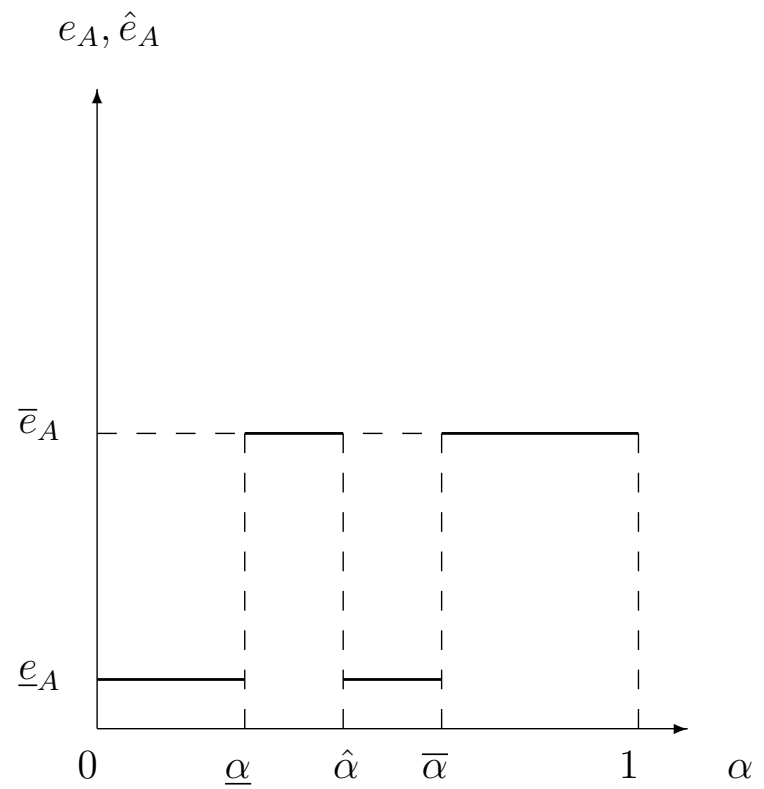


Figure 5(a)

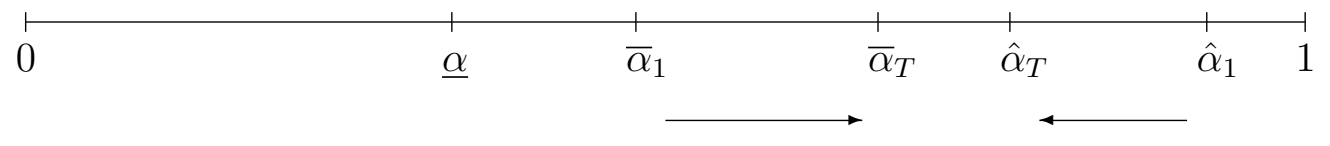

Figure 5(b)

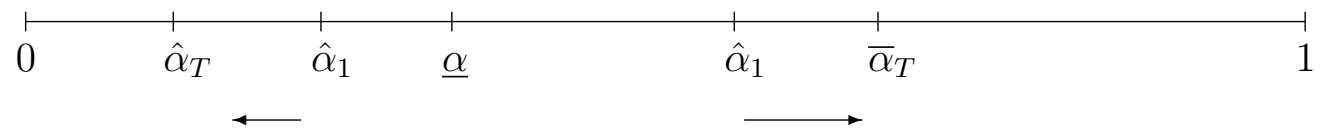

Figure 5(c)

$\begin{array}{llllll}\hat{\alpha}_{T} & \underline{\alpha} & \bar{\alpha}_{1} & \hat{\alpha}_{1} & \bar{\alpha}_{T} & 1 \\ & & & \end{array}$

Figure 5(d)

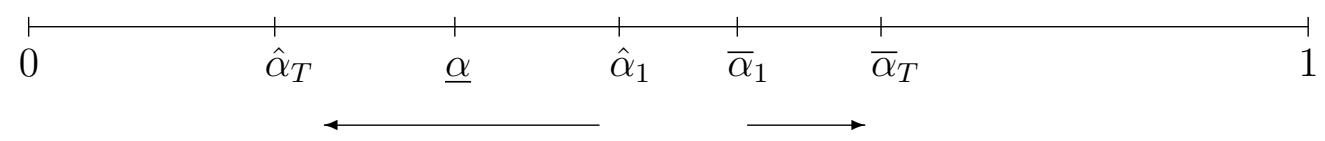

